\title{
158. Behandlungsergebnisse der venösen Thrombektomie mit temporärer A.V.-Fistel bei tiefer Beinvenenthrombose mit und ohne Lungenarterienembolie
}

\author{
M. Edelmann, W. Sandmann, H. Nüllen und H. Kniemeyer \\ Chirurgische Klinik A, Universität Düsseldorf, Moorenstraße 5, D-4000 Düsseldorf
}

Venous Thrombectomy with Temporary Arteriovenous Shunt: Early and Late Results in Deep Venous Thrombosis with or without Pulmonary Emboli

Summary. Eighty-eight patients with deep venous thrombosis (DTV) - 94 thrombosis, 30 with emboli - were treated from 1977 to 1982 by venous thrombectomy with temporary arteriovenous (AV) shunt. The average occlusion time was 5 days $(1-9)$, the sex distribution was similar, and the mean age was 46 years of age $(15-73)$. Postoperatively, the deep venous system was found to be free with functioning venous valves in $83 \%$ of the patients; after 1 year the figure was $81 \%$; and from $1 \frac{1}{2}$ to $2 \frac{1}{2}$ years or longer, it was $86 \%$. No re-emboli were observed. Therefore, early venous thrombectomy (6-8 days) with temporary AV shunt can be regarded as the method of treatment in reopening the occluded deep venous vessels with functioning valves, thus preventing the post-thrombotic syndrome.

Key words: DTV with or without emboli - Venous thrombectomy with AV shunt - Functioning venous valves.

Zusammenfassung. 88 Patienten mit tiefer Beinvenenthrombose, insgesamt 94 Thrombosen, 30 embolisierend, wurden von 1977-1982 einer venösen Thrombektomie mit temporärer A.V.-Fistel unterzogen. Die durchschnittliche Gefäßverschlußzeit betrug 5 Tage (1-9 Tage) bei gleicher Geschlechtsverteilung und einem mittleren Alter von $46(15-73)$ Jahren. Postoperativ war das tiefe Venensystem frei mit intaktem Klappenapparat bei $81 \%$, nach $1 \frac{1}{2}-2 \frac{1}{2}$ Jahren und länger bei $86 \%$ der nachbeobachteten Patienten. Es traten keine erneuten Embolien auf. Die frühzeitige (innerhalb von 1 Woche) venöse Thrombektomie mit temporärer A.V.-Fistel erweist sich als die Behandlungsmethode, die der Forderung nach Wiedereröffnung der venösen Strombahn mit funktionsfähigen Venenklappen am ehesten gerecht wird.

Schliisselwörter: Tiefe Beinvenenthrombose - venöse Thrombektomie mit temporärer A.V.-Fistel - intakter Klappenapparat.

\section{Iatrogene Gefäßverletzungen bei Kindern und Jugendlichen - Nachuntersuchungsergebnisse}

\author{
M. Staudacher, M. Deutsch, M. Schlemmer und M. Wimmer \\ II. Chirurgische Universitäts-Klinik Wien Universitäts-Kinderklinik Wien, Spitalgasse 23, A-1090 Wien
}

\section{Iatrogenic Vascular Lesions in Children and Adults: Results of Follow-up Examinations}

Summary. From 1978 to 1982, 24 iatrogenic vascular lesions, mainly of the arteria femoralis communis, were treated in children (age 4 months to 14 years) by diagnostic or therapeutic measures. According to the lesion, oversewing, thrombectomy, or dilatation of the vessel by a Fogarty-catheter was performed. In some cases microvascular surgery was necessary. Two-thirds of the children underwent a follow-up examination. Immediate operative revision avoided growth shortening of the extremities.

Key words: Iatrogen vascular lesions - Children - Immediate operative revision - No growth shortening.

Zusammenfassung. Von 1978 bis 1982 wurden an der Klinik 24 iatrogene Gefäßverletzungen, vorwiegend der Arteria femoralis communis bei diagnostischen oder therapeutischen Maßnahmen bei Kindern im Alter von 4 Monaten bis 14 Jahren behandelt. Es wurde jeweils, entsprechend der Verletzungsart die Übernähung, Thrombektomie und Dilatation des Gefäßes mit dem Fogarty-Katheter durchgeführt. Vereinzelt wurden auch mikrogefäßchirurgische Maßnahmen notwendig. $2 / 3$ der Kinder konnten nachuntersucht werden. Durch sofortige operative Revision konnten Wachstumsverkürzungen der entsprechenden Extremitäten vermieden werden.

Schliisselwörter: Iatrogene Gefäßverletzung, Kinder - Sofortige Revision - Keine Wachstumsverkürzung. 